Relations industrielles

Industrial Relations

\title{
Le rapport de la Commission Stewart
}

\section{Roger Charbonneau}

Volume 15, numéro 1, janvier 1960

URI : https://id.erudit.org/iderudit/1022073ar

DOI : https://doi.org/10.7202/1022073ar

Aller au sommaire du numéro

\section{Éditeur(s)}

Département des relations industrielles de l’Université Laval

ISSN

0034-379X (imprimé)

1703-8138 (numérique)

Découvrir la revue

Citer ce document

Charbonneau, R. (1960). Le rapport de la Commission Stewart. Relations industrielles / Industrial Relations, 15(1), 115-118.

https://doi.org/10.7202/1022073ar

Tous droits réservés (C) Département des relations industrielles de l’Université Laval, 1960
Ce document est protégé par la loi sur le droit d'auteur. L’utilisation des services d'Érudit (y compris la reproduction) est assujettie à sa politique d'utilisation que vous pouvez consulter en ligne.

https://apropos.erudit.org/fr/usagers/politique-dutilisation/ 


\section{Le rapport de la Commission Stewart}

\section{Roger Charbonneau}

La Commission royale d'enquête sur les écarts de prix des denrées alimentaires, aussi connue sous le nom de Commission Stewart, vient de présenter les deux premiers volumes de son rapport.

Il s'agit d'un travail considérable, bourré de chiffres, renfermant beaucoup de détails précis sur des techniques commerciales et aussi des considérations économiques d'un grand intérêt. L'enquête a été menée à travers le pays et les commissaires ont en outre puisé largement aux statistiques publiées ou inédites des divers ministères fédéraux. Ils ont également envoyé un questionnaire à des industries et des commerces alimentaires. Le travail porte sur les produits de l'agriculture et de la pêche. Etant donné l'espace limité à ma disposition, je me limiterai à l'analyse de cette partie du rapport qui traite des produits agricoles.

La Commission a posé comme suit le problème de fond: en prenant les chiffres de 1949 comme base, l'indice des prix payés par les consommateurs avait augmenté de $20 \%$ à la fin de 1958 alors que celui des prix obtenus par le fermier était resté stable; quelles sont les causes de cette divergence? Autrement dit, la Commission n'a pas voulu établir les causes fondamentales de l'écart des prix: elle s'est contentée de rechercher les raisons pour lesquelles l'écart avait augmenté. Le rapport indique que la carence de statistiques a empêché la Commission de compléter la tâche qui lui avait été expressément confiée par le Gouvernement. On doit le regretter, parce que le travail tel que présenté ne permet pas de tirer de conclusions suffisamment fondées sur bien des points et que les groupes intéressés profiteront, comme d'habitude, de données incomplètes pour défendre leur opinion.

La Commission s'est d'abord attachée aux causes qui influent sur le prix de détail des produits agricoles. Elle a mis en valeur le fait que les Canadiens, dont le revenu réel a augmenté plus vite que leur consommation de denrées alimentaires durant la décade 1949-1958, étaient déjà des gens bien nourris au début de cette même décade et que dans un pareil cas, les revenus en excédent sont utilisés pour d'autres fins que l'alimentation. Par ailleurs, en gens plus aisés, ils ont été portés à payer plus cher pour les services qui accompagnent la distribution des produits alimentaires (présentation, emballage, choix abondant, magasins à l'atmosphère agréable, stationnement facile). Le rapport admet cependant qu'il est malheureusement impossible d'établir par les statistiques la part du produit et celle des services dans le prix payé par le consommateur.

La Commission s'est ensuite préoccupée du prix reçu par le producteur et elle a conclu que le comportement des prix des produits 
agricoles sur les marchés mondiaux a eu une grande influence sur les prix intérieurs. L'augmentation de la productivité amenée par la mécanisation a aussi contribué à déprimer les prix.

Après avoir établi les deux points extrêmes du problème, la Commission s'est attaquée à l'étude de toute la chaîne intermédiaire: assemblage et transformation des produits, commerce de gros et de détail.

Elle a d'abord noté, en y attachant une grande importance, la domination du commerce de détail par les épiceries géantes. Elle a également souligné que les magasins à succursales jouent aussi le rôle de grossistes et que les grossistes commanditent les groupes de magasins syndiqués.

Toụt en louant les remarquables méthodes de vente et d'administration des magasins à succursales, la Commission indique que les services rendus ont augmenté les prix au détail. Dans ses conclusions, elle admet d'abord que les services étaient désirés par le consommateur, mais elle affirme par ailleurs qu'une partie importante des dépenses engagées pour stimuler les ventes constitue un gaspillage.

La Commission paraît croire que les épiciers indépendants ont des chances de survie, mais elle souligne qu'il leur faudra «beaucoup de compétence et d'habileté ». De mềme, la situation du grossiste qui n'a pas établi de liens avec des groupes de détaillants est devenue plus précaire.

Enfin, la concentration dans le commerce de gros et de détail a eu pour parallèle une concentration dans l'industrie de transformation.

Dans l'ensemble, la concentration dans le commerce de détail exercerait une pression à la baisse sur les prix de l'industrie de transformation. Plusieurs associations agricoles ont représenté à la Commission, que les industriels ont été ainsi amenés à abaisser les prix auxquels ils achètent des fermiers. Le rapport indique cependant que ce fait n'a pas été nettement prouvé et que là où une coopérative de fermiers opère la transformation du produit, l'écart du prix au producteur et du prix au consommateur n'a pas diminué.

Le rapport étudie longuement les méthodes de vente des magasins à succursales. Après avoir noté que leur part du marché de l'épicerie est passée de $32 \%$ à $44 \%$ au cours de la dernière décade, il souligne que ces progrès sont dus à la vision des administrateurs de ces entreprises qui ont prévu le développement des banlieues résidentielles, laocroissement du nombre des automobiles et les goûts nouveaux créés chez le consommateur par l'accroissement de son revenu. Mais la Commission reproche à ces détaillants de n'avoir pas utilisé la coupe de prix comme instrument systématique de vente.

Après tous ces préambules, la Commission s'est attaquée à l'étude des frais de tous les intermédiaires entre le fermier et le consomma- 
teur. Elle a d'abord noté l'accroissement des marges brutes des do taillants et des industriels. De plus, elle a relevé que le salaire hebdomadaire dans les magasins à succursales et les établissements de transformation a augmenté de 50\% depuis 1949 . Elle a enfin établi que la marge de bénéfice des entreprises en relation avec le capital engagé ne semble pas exagérée.

Quant aux frais de vente, la Commission s'est attachée à quatre d'entre eux: le transport, la réclame, l'entreposage frigorifique et l'empaquetage. Elle y a consacré une partie importante de son rapport et s'est livrée, particulièrement dans le cas de la réclame, à des spéculations économiques et sociales fort élaborées.

J'avoue avoir été un peu surpris de cette insistance. En effet, les frais de transport ne représentent que $11 \%$ du total des frais de vente, la publicité, seulement $5 \%$, les frais d'entreposage, moins de $1 \%$ et les fournitures d'emballage, $10 \%$. On a fait état de l'augmentation de ces frais durant la décade 1949-1959. Les hausses ont beaucoup varié, d'un maximum de $400 \%$ pour la publicité jusqu'à un minimum de $76 \%$ pour le transport. Il faut tout de même considérer que l'augmentation des affaires qui ont plus que doublé justifie une certaine augmentation de publicité. Quant aux frais d'entreposage, la popularité récente des aliments congelés en explique facilement l'accroissement. Enfin, la Commission elle-même note que ceux qui lui ont exprimé leurs vues au sujet des frais d'emballage ont attaché trop d'importance relative à cette question. Il ne faut pas en effet oublier le revers de la médaille: réduction du gaspillage, simplification de la manutention et maintien de la qualité.

Mais mon étonnement a grandi en lisant les recommandations de la Commission ou tout au moins, dans certains cas, le ton employé. Ainsi le rapport Stewart suggère une enquête, en vertu de la Loi des enquêtes sur les coalitions, sur les méthodes d'achat et de vente des grandes entreprises commerciales dans les industries alimentaires. Il est difficile de voir comment la Commisson en est arrivée à la conclusion d'une telle nécessité. La Commission réclame également la détermination de ce qu'est une bénéfice juste et raisonnable par rapport au capital investi et recommande de relever l'impôt sur le revenu en excédent de ce qui paraît raisonnable. Cette conclusion est d'autant plus surprenante que, sauf dans deux cas isolés, les tableaux du rendement sur le capital compilés par la Commission révèlent, à travers les fluctuations annuelles, une tendance à la baisse entre 1949 et 1958. Le rapport dit même que « d'une façon générale, les bénéfices provenant de la transformation et de la distribution des aliments se comparent défavorablement aux taux d'autres secteurs de l'industries (Vol. 1, p. 45).

La Commission insiste sur le fait qu'il arrive parfois que les industries posent des gestes au détriment des consommateurs en général. 
Elle en déduit que ce fait devrait généralement être reconnu et admis par l'industrie, et que s'il ne l'était pas, il faudrait que l'Etat intervienne. Ce langage me paraît un peu étonnant quand on l'accouple aux compliments prodigués à l'industrie dans le rapport.

D’autres conclusions sont beaucoup mieux amenées. Par exemple, le rapport suggère l'établissement d'un conseil permanent qui surveillerait prix, productivité et profits dans l'industrie alimentaire et présenterait un rapport annuel. Il recommande aussi que, si les bons-primes et timbres-primes survivent, le client ait le droit de se faire rembourser en espèces par le détaillant, de façon à obtenir une ristourne. Il demande l'établissement d'un office fédéral destiné à renseigner le consommateur sur les lois et les services qui le protègent. En vue d'aider le petit épicier, les commissaires désirent que l'on recueille et mette à sa disposition des renseignements détaillés sur l'administration des petites entreprises. Pour aider les fermiers, il faudrait adopter une loi fédérale prévoyant la constitution en compagnies des coopératives. Enfin, le rapport insiste sur le besoin de recueillir plus de statistiques.

Somme toute, personne n'est oublié, pour son bonheur ou pour son malheur.

En conclusion générale, je dirai que la Commission Stewart a fait un gros travail de déblaiement dans une question complexe et que les plus intéressantes recommandations sont justement celles qui visent à mener à bonne fin une oeuvre si bien commencée.

\section{Le contrôle des monopoles}

Quelques commentaires en marge du bill C-59

\section{Gérald Marion}

L'année dernière, le gouvernement fédéral avait distribué à la Chambre des Communes le bill C-59 dont l'objet est d'apporter des changements substantiels à la législation anti-trust existante. A la suite de protestations, le bill fut retiré avant qu'il ait fait l'objet de débats parlementaires. On peut s'attendre, cependant, qu'au cours de la prochaine session, le gouvernement revienne à la charge avec un nouveau projet. C'est pourquoi il n'est pas inutile de faire une analyse de l'ancien projet de loi.

\section{LA LOI ACTUELLE}

Selon la présente loi des enquêtes sur les coalitions et son interprétation dans la jurisprudence, toute entente commerciale ou industrielle est illégale parce qu'elle est une menace à l'intérêt public. Le lien qui 